\title{
Septal pore apparatus of the smut Ustacystis waldsteiniae ${ }^{1}$
}

\section{Robert Bauer}

Universität Tübingen, Institut für Biologie I, Lehrstuhl Spezielle Botanik und Mykologie, Auf der Morgenstelle I, D-72076 Tübingen, Germany

Kurt Mendgen

Universität Konstanz, Fakultät für Biologie, Lehrstuhl für Phytopathologie, Universitätsstr. 10, D.78434

Konstanz, Germany

Franz Oberwinkler

Universität Tübingen, Institut für Biologie I, Lehrstuhl Spezielle Botanik und Mykologie, Auf der Morgenstelle 1, D-72076 Tübingen, Germany

Abstract: The septal pore apparatus in intercellular hyphae of Ustacystis waldsteiniae was analyzed by serialsection electron microscopy using chemically fixed and high pressure frozen/freeze substituted samples. Septa have a central "simple" pore with rounded, nonswollen margins. However, the pore apparatus is a highly complex structure, differing significantly from that found in "simple"- and complex-septate basidiomycetes. Three features are typical: 1) the pore canal is traversed by two symmetrically arranged bands, 2) a dome shaped membranous cap, intimately attached to the plasma membrane, encloses the pore on both sides, and 3) a thin electron transparent layer and a layer of medium electron density cover the cytoplasmic side of the membranous cap. From our observations of septal pore morphology we suggest a general absence of transport of organelles including ribosomes through the pore.

Key Words: freeze substitution, septal pores, smuts, Ustacystis waldsteiniae

\section{INTRODUCTION}

Evolutionary trends within the smuts and between the smuts and other heterobasidiomycetous groups are only poorly understood (Oberwinkler, r985; Vánky, 1987). Therefore, for the last $8 \mathrm{yr}$ we have examined numerous smuts and presumed relatives by light and electron microscopy.

'Part 97 of the series "Studies in Heterobasidiomycetes." Accepted for publication September 1, 1994.
Septal pore morphology studied in many basidiomycetes (see Oberwinkler, 1985 and the references therein) continues to play an important role in the arrangement of these taxa (Wells, 1994). These studies indicate that characteristics of septal pore apparatus are conserved and indicative for natural relationships. Perhaps because many smuts are difficult to collect in a serviceable condition for electron microscopy and/ or difficult to fix, few studies of their septal pore characteristics are available (Oberwinkler, 1985 ; Bauer et al., 1989). Here, we describe septal pore architecture of Ustacystis waldsteiniae (Peck) Zundel based on detailed observations mainly of samples prepared by use of high pressure freezing and freeze substitution.

\section{MATERIALS AND METHODS}

Materials used.-Leaves of Waldsteinia geoides Willd. with young sori of Ustacystis waldsteiniae were collected in a natural area of the Botanical Garden of the Universität Tübingen (Tübingen, Baden-Württemberg, Germany, 14 April 1992, designated $R$. Bauer 1056) where no fungicides are used.

Conventional chemical fixation. - Infected areas of leaves were fixed in $2 \%$ glutaraldehyde in $0.1 \mathrm{M}$ sodium cacodylate buffer $(\mathrm{pH} 7.2)$ at room temperature overnight. Following six transfers in $0.1 \mathrm{M}$ sodium cacodylate buffer, samples were postfixed in $1 \%$ osmium tetroxide in the same buffer for $2 \mathrm{~h}$ in the dark, washed in distilled water, and stained in $1 \%$ aqueous uranyl acetate for $1 \mathrm{~h}$ in the dark. After five washes in distilled water, samples were dehydrated in acetone, using 10 min changes at $25 \%, 50 \%, 70 \%, 95 \%$, and 3 times in $100 \%$ acetone. Samples were embedded in Spurr's plastic (Spurr, 1969).

Serial sections $(65-75 \mathrm{~nm})$ were cut on a ReichertJung Ultracut E (Leica, Nußloch) with a diamond knife. Sections were mounted on formvar-coated, single-slot copper grids, stained with lead citrate (Reynolds, 1963) at room temperature for 3-5 min, and washed again with distilled water. They were examined with a transmission electron microscope EM 109 (Zeiss, Oberkochen) at $80 \mathrm{kV}$.

High pressure freezing and freeze substitution.-Infected areas of leaves were removed with a 2-mm cork borer. In order to remove air from intercellular spaces, sam- 
ples were infiltrated with distilled water containing $6 \%$ $(\mathrm{v} / \mathrm{v})(2.5 \mathrm{M})$ methanol for approximately $5 \mathrm{~min}$ at room temperature. Single samples were placed in an aluminum holder (one half with a hollow of $0.3-\mathrm{mm}$ depth for the sample and the other a flat top) and frozen immediately in the high pressure freezer HPM 010 (Balzers Union, Lichtenstein) as described in detail by Mendgen et al. (I99I).

Substitution medium ( $1.5 \mathrm{ml}$ per specimen) consisted of $2 \%$ osmium tetroxide in acetone which had been dried over calcium chloride. Freeze substitution was performed at $-90 \mathrm{C},-60 \mathrm{C}$, and $-30 \mathrm{C}, 8 \mathrm{~h}$ for each step, using a Balzers freeze substitution apparatus FSU 010. The temperature was then raised to approximately $0 \mathrm{C}$ over a 30 -min period and samples were washed in dry acetone for another $30 \mathrm{~min}$. Infiltration with an Epon/Araldite mixture (Welter et al., 1988) was performed stepwise: $30 \%$ resin in acetone at $4 \mathrm{C}$ for $7 \mathrm{~h}, 70 \%$ and $100 \%$ resin at $8 \mathrm{C}$ for $20 \mathrm{~h}$ each and $100 \%$ resin at $18 \mathrm{C}$ for approximately $12 \mathrm{~h}$. Samples were then transferred to fresh medium and polymerized at $60 \mathrm{C}$ for $10 \mathrm{~h}$.

Samples were then processed as for chemically fixed samples described above except that the sections were additionally stained with $1 \%$ aqueous uranyl acetate for $1 \mathrm{~h}$.

Septal pores investigated.--Data for this report were obtained from 45 septal pores prepared by high pressure freezing and freeze substitution, and 28 prepared by chemical fixation. Septal pores were investigated from hyphae located in peripheral and central parts of the sori.

\section{RESULTS}

Examples of septal pores prepared by high pressure freezing followed by freeze substitution or conventional chemical fixation are shown in Figs. 1-4 and FIGS. 5, 6, respectively. By both fixation techniques the general septal pore architecture was recognizable. In high pressure frozen hyphae, however, septal pore morphology had a more regular appearance and was more distinct than after conventional fixation (compare FIGS, 1, 2 with FiGs. 5, 6). Thus, in high pressure frozen hyphae plasma membrane followed the contour of the septum closely (FIGS. 1-4), whereas in conventionally fixed hyphae plasma membrane was often irregularly folded (FIGS. 5, 6). In addition, plasma membrane of a high pressure frozen cell appeared different from that after conventional fixation. In conventionally fixed cells (FIGs. 5, 6), the two electron dense layers of the tripartite plasma membrane were more or less equal in thickness. In high pressure frozen cells, however (FIGS. 1-4), the exoplasmic electron dense layer of plasma membrane appeared thinner than the other. Septal walls were usually of greater electron density after high pressure freezing than after conventional fixation. Moreover, the trilamellate nature of septa appeared clearer after high pressure freezing than after conventional fixation (compare FIG. 4 with FIG. 5), and the septal wall often appeared irregularly swollen after conventional fixation (FIGs. 5, 6). Each septum had a central "simple" pore with nonswollen margin. The pore lip after high pressure freezing, but not after conventional fixation, was generally rounded (compare FIGs. 1-4 with FIgs. 5, 6). Plasma membrane was continuous through the pore and the diameter of the intermembrane space within the pore was 0.06 $0.07 \mu \mathrm{m}$.

The pore apparatus was a highly complex structure. The pore canal was traversed by two symmetrically arranged electron dense bands (labelled in FIG. 1). Sometimes, these bands showed a partial fibrillar substructure after high pressure freezing (FIG. 2). They contacted plasma membrane in the pore. In three pores with extremely thin cross walls these bands were absent (not illustrated), apparently reflecting different developmental stages. Outside these bands towards the cytoplasm a dome shaped cap limited the orifice area of the pore at either side (labelled in FIG. 1). In both high pressure frozen (FIGs. 1-4) and conventionally fixed (FIGS. 5, 6) septa, the membranous nature of these caps was apparent: two electron dense layers were separated by a thin electron transparent layer. The two electron dense layers were equal in thickness (FIGS. 1-6). Layering of the cap was similar to that of plasma membrane after conventional fixation (FIGS. 5,6 ), whereas after high pressure freezing the electron dense layer of plasma membrane at the exoplasmic face appeared thinner than each of the electron dense layers of the cap (FIGS. 1-4). In high pressure frozen hyphae, however, layering of caps was basically similar to that of vacuole membranes (FIG. 4). The cap was unfenestrated. It terminated directly next to the adjacent electron dense band in the protoplasmic electron dense layer of plasma membrane and was never observed extending into the exoplasmic electron dense layer of plasma membrane (FIGS. 1-4). Under optimal sectioning conditions it appeared that at the contact area the protoplasmic electron dense layer of plasma membrane was continuous with the two electron dense layers of the cap, extending at a right-angle to the cap (FIG. 3). Occasionally, small lines of medium electron density extended between the two caps of a pore traversing the inner bands (FIG. 2). A thin electron transparent layer and a more or less diffuse layer of medium electron density followed the contour of the cap at the cytoplasmic side (labelled in Fig. 1). Cisternae of endoplasmic reticulum were often associated with the 


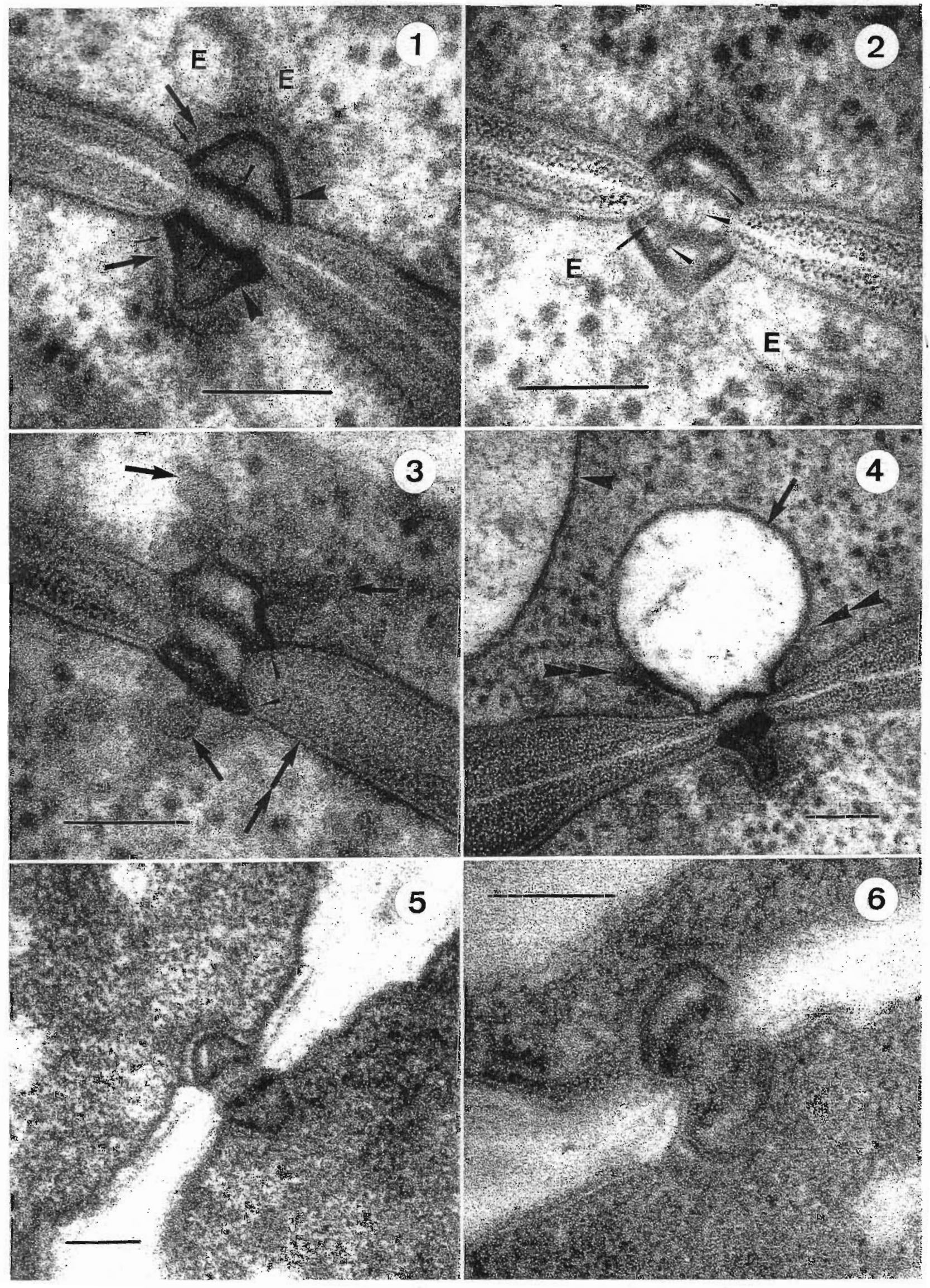


laver of medium electron density (FIGs. 1, 2). They were of poor contrast after high pressure freezing and conventional fixation. No ribosomes or other organelles were observed in the pore domain enclosed by caps.

Usually the two caps of a pore were smooth and svmmetrically arranged. In seven high pressure frozen pores, however, one or several bulges were present either at one cap or at both (FIG. 3), extending from the cap into the cytoplasm. In addition, in six high pressure frozen pores one cap was extremely expanded in a sac-like fashion thus giving the impression of a vacuole (FIG. 4). In these cases the layer of medium electron density surrounded only the basal part of the cap adjacent to the septal wall and the membranous nature of the cap appeared clearly. Such modifications of the caps were not observed in conventionally fixed samples.

\section{DISCUSSION}

Septal pore fine structure of $U$. waldsteiniae after high pressure freezing is very similar to that after conventional fixation. The differences between the high pressure frozen and conventionally fixed septal pores observed in this study reflect only the general differences between freeze substitution and chemical fixation, as described and illustrated by many authors (e.g., Hoch and Howard, 1980, 1981; Hoch and Staples, r983; Hoch, 1986; Berbee and Wells, 1988; Mims et al., 1988; Welter et al., 1988; Berbee et al., I99r; Lü and McLaughlin, 1991). Thus, the comparison of high pressure frozen and conventionally fixed septal pores indicate that use of $6 \%$ methanol as a cryoprotectant did not influence septal pore fine structure.

Paradox of the "membrane" cap.--The different biomembranes observed in this study including the caps share a common substructure. In addition, differences observed in layering among the biomembranes are similar to those between caps on the one hand and biomembranes on the other. For example, differences in layering between plasma membrane and the vacuole membrane are comparable to those between the cap and vacuole membrane. These data suggest that the caps enclosing pores are composed of single biomembranes. This situation is extremely unusual and presents somewhat of a paradox. Biomembranes are endless, being in section always arranged in rings. This dogma (Kleinig and Sitte, I992) supports our observation that caps are continuous with the protoplasmic layer of plasma membrane. However, another dogma says that single biomembranes are unbranched (Kleinig and Sitte, 1992). Only if two membranes fuse or vice versa (for example secretory vesicle/plasma membrane fusion or vesicle production of dictyosomes), can a situation develop in which biomembranes are branched, similar to that of $U$. waldsteiniae. But this is a dynamic process and is not stable as in the septal pores of $U$. waldsteiniae. For the situation in $U$. waldsteiniae there are two explanations: 1) the caps are not composed of biomembranes, or 2) they are composed of biomembranes, in which case the biomembrane is unusually branched or two biomembranes are unusually fused.

Functional aspects.--Septal pores play a dominant role in communication between hyphal cells. They allow cytoplasmic continuity between cells and control cytoplasmic streaming (Bracker, 1967). Cytoplasmic streaming is regarded by Bracker and Butler (1964) as an important mechanism for nutrient transport and cytoplasmic translocation. For dolipores with parenthesomes several authors have suggested that communication between hyphal cells is regulated not only by the pore itself, but also by the parenthesomes and other material in the pore canal, e.g., bands or rings (Butler and Bracker, 1970; Thielke, 1972; Lü and McLaughlin, 1991). Bracker and Butler (1964) and Tu et al. (1977) suggested that organelles can pass through

Figs. 1-6. Septal pore apparatus of Ustacystis waldsteiniae prepared by high pressure freezing and freeze substitution (FIGs. 1-4), or chemical fixation (Fics. 5, 6). Bars $=0.1 \mu \mathrm{m}$. 1. Typical septal pore apparatus after high pressure freezing with rounded pore margin, two electron dense bands (small arrowheads) and two tripartite membranous caps (large arrowheads). A thin electron transparent layer (small arrows) and a more diffuse layer of medium electron density (large arrows) follow contours of the membranous caps at each cytoplasmic side. Cisternae of endoplasmic reticulum (E) contact the layer of medium electron density at one side. Note unequal layering of the plasma membrane. 2. One band shows partial fibrillar substructure (arrow). Note that thin lines extend between membranous caps (arrowheads). E, associated cisternae of endoplasmic reticulum. 3. Section showing membranous caps with three bulges (arrows) and unequal layering of plasma membrane. The protoplasmic electron dense layer of plasma membrane (double arrow) appears continuous with two electron dense layers of membranous caps, extending at a right-angle (arrowheads). 4. One membranous cap is highly expanded (arrow) and the layer of medium electron density (double arrowheads) surrounds only the basal part of this cap adjacent to the septal wall. Note that layering of this cap (arrow) is basically similar to that of neighbouring vacuole membrane (arrowhead). 5 , 6 . Two typical septal pore apparatus after conventional fixation showing irregular plasma membrane, but the general septal pore architecture is comparable to that after high pressure freezing (labelled in Fic. 1). Note that the membranous nature of caps is clearly recognizable. 
the pores in Rhizoctonia spp. because of the wide perforations in parenthesomes and pores.

It is clear from our study that in $U$. waldsteiniae organelles including ribosomes cannot pass through intact septal pores. Two membranous caps and at least four additional structures may prevent passage of organelles. This interpretation is supported by the fact that the septal pore domain enclosed by caps is wholly organelle-free. But nutrient transport through the pore must be possible, at least from haustoria to intercellular hyphae, and finally to the teliospore initials. The six structures observed in septal pores of $U$. waldsteiniae may also serve as filters to prevent uncontrolled transport of substances from cell to cell. It is unclear, whether modifications in pore caps observed in this study after high pressure freezing represent real transfer stages because the possibility of artifacts cannot be excluded. Thus, expansion of membranous caps apparently involves the rupture of the surrounding layer of medium electron density.

Phylogenetic aspects. - As reviewed by Khan and Kimbrough (1982) and Oberwinkler (1985), basidiomycetes possess two main types of septal pore apparatus: "simple" pores and dolipores. Dolipores possess pore swellings either without pore caps or with cupulate, nonperforate or perforate pore caps. "Simple" pores lack the pore swelling and may or may not be associated with specialized structures.

Several distinct types of simple septal pores may be distinguished in auricularioid fungi: 1) Cryptomycocolax-type in Cryptomycocolacales (Oberwinker and Bauer, 1990): the pore possesses a pulley-wheel pore occlusion. An organelle-free zone, delimited by microbodies and Woronin bodies surrounds each side of the septal pore. These septal pores closely resemble septal pores of Uredinales and of many ascomycetes. 2) Rust-type in the rusts (Littlefield and Heath, 1979) and auricularioid phytoparasites except Kriegeria eriophori Bres. (Bourett and McLaughlin, 1986; Boehm and McLaughlin, 1989; Bauer and Oberwinkler, 1994): septal pores possess characteristic pulley-wheel plugs. The septal pore area consists of an organelle free zone surrounded by a circular arrangement of microbodies. Rust septal pores closely resemble Cryptomycocolax septal pores, but differ in the absence of Woronin bodies. 3). Agaricostilbum-type in Agaricostilbales (Oberwinkler and Bauer, 1989): septal pores are associated with electron dense nonmembrane-bounded globules. 4) Atractiella-trpe in Atractiellales sensu Oberwinkler and Bauer (1989): pores are associated with globose compartments differentiated into electron dense peripheral lavers and electron transparent central parts. 5) Stilinum-tipe in Chionosphaeraceae and Colacogloea pen: q, horat (Bourd. \& Galz.) Oberw. \& Bandoni (Ober- winkler and Bauer, 1989; Oberwinkler et al., I99oa): small septal pores are not associated with vesicles or vesicle-like structures. 6) Kriegeria-type in Kriegeria eriophori (Doublés and McLaughlin, 1991): pore morphology is essentially identical to that of the Stilbumtype, but septa are multiperforate. 7) Atractogloea-type in Atractogloeaceae (Oberwinkler and Bauer, 1989): a swollen septal wall is more or less abruptly flattened towards the pore margin. Pores are not associated with electron dense vesicles or bodies. 8) Septobasidium-type in Septobasidiales and Platygloea disciformis (Fr.) Neuhoff (Oberwinkler et al., 1990a): pore morphology is essentially identical to that of Atractogloea stillata $\mathrm{Ob}$ erw. \& Bandoni, but the pores are associated with electron dense globules and bandings. 9) Heterogastridium-type in Heterogastridiales (Oberwinkler et al., I990b): the periphery of electron dense bodies, adjacent to the septal pore, is not sharply delimited from the cytoplasm. Electron dense bodies then fuse and occlude septal pores. Septal pore apparatus of $U$. waldsteiniae differs significantly from that found in "simple"-septate auricularioid basidiomycetes. Presence of two bands and two membranous caps at the pore in $U$. waldsteiniae reflects a fundamental difference from "simple"-septate auricularioid basidiomycetes.

Members of holobasidiate Exobasidiales (Khan et al., 1981) and Graphiolales (Oberwinkler et al., 1982) also revealed a "simple" septal pore structure with a more or less rounded pore margin. Perhaps the membranous structures sometimes found enclosing septal pores of Exobasidium vaccinii Wor. and E. camelliae Shirai at one or both sides (Khan et al., I98I) are homologous to membranous caps enclosing septal pores of $U$. waldsteiniae, but additional studies are needed to clarify this supposition.

Septal pores in smuts are still insufficiently examined. Moore (1972), Robb (1972), Deml (1977), Bauer et al. (1989) and Boekhout et al. (1992) found no pores in many smuts; however, narrow pores with slightly inflated pore margins were found in basidial septa of Ustilago maydis (De Candolle) Corda (Ramberg and McLaughlin, 1980; O'Donnell and McLaughlin, 1984), $U$. tritici (Pers.) Rost., $U$. avenae (Pers.) Rost. (Bauer et al., 1989) and $U$. esculenta $\mathrm{P}$. Hennings (Nagler et al., 1990). Dolipores in hyphae were described and illustrated for Entorrhiza casparyana (P. Magnus) Lagerheim (Deml and Oberwinkler, 198I) and some species of Tilletia (Deml, 1977; Roberson and Luttrell, 1989), whereas distinct "simple" pores were described for Urocystis spp. (Nagler, 1987). The septal pore apparatus of $U$. waldsteiniae resembles that of species of Urocystis. Based on morphological similarities, Ustacystis waldsteiniae was originally described as Urocystis waldsteiniae Peck. Zundel (1945) transferred the species to the new genus Ustacystis, based on presence of 
phragmobasidia. Obviously, within smuts, a monophyletic group exists with different basidial types.

\section{ACKNOWLEDGMENTS}

We thank Annette Klink and Sabine Silberhorn for excellent technical assistance and the Deutsche Forschungsgemeinschaft for financial support.

\section{LITERATURE CITED}

Bauer, R, and F. Oberwinkler. 1994. Meiosis, septal pore architecture and systematic position of the heterobasidiomycetous fern parasite Herpobasidium filicinum. Canad. J. Bot. 72: 1229-1242.

- - - $\longrightarrow$, and G. Deml. 1989. Ultrastruktur der Basidiensepten phragmobasidialer Brandpilze. Z. Mykol. 55: 163-168.

Berbee, M. L., R. Bauer, and F. Oberwinkler. 1991. The spindle pole body cycle, meiosis, and basidial cytology of the smut fungus Microbotryam violaceum. Canad. J. Bot. 69: 1795-1803.

tosis and septal pore apparatus in Tremella globospora. Mycologia 80; 479-492.

Boehm, E. W. A., and D. J. McLaughlin. 1989. Phylogeny and ultrastructure in Eocronartium muscicola: meiosis and basidial development. Mycologia 81: 98-114.

Boekhout, T., Y. Yamada, A. C. M. Weijman, H. J. Roeymans, and W. H. Batenburg-van der Vegte. 1992. The significance of coenzyme $Q$, carbohydrate composition and ultrastructure for the taxonomy of ballistoconidia forming yeasts and fungi. Syst. Appl. Microbiol. 15: 110.

Bourett, T. M., and D. J. McLaughlin. 1986. Mitosis and septum formation in the basidiomycete Helicobasidium mompa. Canad. J. Bot. 64: 130-145.

Bracker, C. E. 1967. Ultrastructure of fungi. Annual Rev. Phytopathol. 5: 343-374.

- , and E. E. Butler. 1964. Function of the septal pore apparatus in Rhizoctomia solani. Mycologia 55: 35-58.

Butler, E. E., and C. E. Bracker. 1970. Morphology and cytology of Rhizoctonia solani. Pp. 32-51. In: Rhizoctonia solani: biology and pathology. Ed., J. R. Parmeter, Jr. Univ. of California Press, Berkeley.

Deml, G. 1977. Feinstrukturelle Merkmalsanalysen an Ustilaginales-Arten. Z. Pilzk. 43: 291-303.

- and F. Oberwinkler. 1981. Investigations on Entorrhiza casparyana by light and electron microscopy. Mycologia 73: 392-398.

Doublés, J. C., and D. J. McLaughlin. 1991. A new basidiomycetous septal type: the multiperforate septum in Kriegeria eriophori. Amer. J. Bot. 78: 1542-1548.

Hoch, H. C. 1986. Freeze substitution of fungi. Pp. 183212. In: Ulirastructure techniques for microorganisms. Eds., H. C. Aldrich and W. J. Todd. Plenum Publ. Corp., New York.

$\longrightarrow$, and R. J. Howard. 1980. Ultrastructure of freeze- substituted hyphae of the basidiomycete Laetisaria arvalis. Protoplasma 103: 281--297.

—, and - 1 1981. Conventional chemical fixations induce artifactual swelling of dolipore septa. Exp. Mycol. 5: $167-172$.

zation of the nondifferentiated uredospore germling of Uromyces phaseoli variety typica. Mycologia 75: 795-824.

Khan, S. R., and J. W. Kimbrough. 1982. A reevaluation of the basidiomycetes based upon septal and basidial structure. Mycotaxon 15: 103-120.

,-- , and C. W. Mims. 1981. Septal ultrastructure and the taxonomy of Exobasidium. Canad. J. Bot. 59: 2450-2457.

Kleinig, H., and P. Sitte. 1992. Zellbiologie. 3th ed. Gustav Fischer Verlag, Stuttgart, Jena, Germany and New York.

Littlefield, L. J., and M. C. Heath. 1979. Ultrastructure of rust fungi. Academic Press, New York. ,

Lü, H., and D. J. McLaughlin. 1991. Ultrastructure of the septal pore apparatus and early septum initiation in Auricularia auricula-judae. Mycologia 83: 322-334.

Mendgen, K., K. Welter, F. Scheffold, and G. Knauf-Beiter. 1991. High pressure freezing of rust infected plant leaves. Pp. 31-42. In: Electron microscopy of plant pathogens. Eds., K. Mendgen and D. E. Lesemann. Springer Verlag, Heidelberg, Germany.

Mims, C. W., R. W. Roberson, and E. A. Richardson. 1988. Ultrastructure of freeze-substituted and chemically fixed basidiospores of Gymnosporangium juniperi-virginianae. Mycologia 80: 356-364.

Moore, R. T. 1972. Ustomycota, a new division of higher fungi. Antonie van Leeuwenhoek J. Microbiol. Serol. 38: 567-584.

Nagler, A. 1987. Urocystis Rabenhorst und Ginanniella Ciferri-zwei eigenständige Gattungen? Urocystis galanthi Pape and Ginanniella primulae (Rostr.) Cifferri. Z. Mykol. 53: 331-354.

- , R. Bauer, F. Oberwinkler, and J. Tschen. 1990. Basidial development, spindle pole body, septal pore, and host-parasite-interaction in Ustilago esculenta. Nordic J. Bot. 10:457-464.

Oberwinkler, F. 1985. Zur Evolution und Systematik der Basidiomyceten. Bot. Jahrb. Syst. 107: 541-580.

-, R. J. Bandoni, P. Blanz, G. Deml, and L. KisimovaHorovitz. 1982. Graphiolales: basidiomycetes parasitic on palms. Pl. Syst. Evol. 140: 251-277.

—, and R. Bauer. 1989. The systematics of gastroid, auricularioid heterobasidiomycetes. Sydowia 41: 224256.

— and - 1990. Cryptomycocolax: a new mycoparasitic heterobasidiomycete. Mycologia 82: 671-692.

- - - and R. J. Bandoni. 1990a. Colacogloea: a new genus in the auricularioid heterobasidiomycetes. Canad. J. Bot. 68: 245-255.

— — new order of basidiomycetes. Mycologia 82: 48-58.

O'Donnell, K. L., and D. J. McLaughlin. 1984. Postmeiotic mitosis, basidiospore development, and septation in $U s$ tilago maydis. Mycologia 76: 486-502. 
Ramberg, J. E., and D. J. McLaughlin. 1980. Ultrastructural study of promycelial development and basidiospore initiation in Ustilago maydis. Canad. J. Bot. 58: 1548-1561.

Reynolds, E. S. 1963. The use of lead citrate at high $\mathrm{pH}$ as an electron opaque stain in electron microscopy. $J$. Cell Biol. 17: 208-212.

Robb, J. 1972. Ultrastructure of Ustilago hordei (Pers.) Lagerh. II. Septation in the metabasidium. Canad. J. Genet. Cytol. 14: 839-849.

Roberson, R. W., and E. S. Luttrell. 1989. Dolipore septa in Tilletia. Mycologia 81: 650-652.

Spurr, A. R. 1969. A low-viscosity epoxy resin embedding medium for electron microscopy. J. Ultrastruct. Res. 26: $31-43$.
Thielke, C. 1972. New investigation on the fine structure of mushrooms. Mushroom Sci. 8: 285-293.

Tu, C. C., J. W. Kimbrough, and H. C. Aldrich. 1977. Cytology and ultrastructure of Thanatephorus cucumeris and related taxa of the Rhizoctonia complex. Canad. J. Bot. 55: 2419-2436.

Vánky, K. 1987. Illustrated genera of smut fungi. Crylog. Stud. 1: 1-159.

Wells, K. 1994. Jelly fungi, then and now. Mycologia 86: $18-48$.

Welter, K., M. Müller, and K. Mendgen. 1988. The hyphae of Uromyces appendiculatus within the leaf tissue after high-pressure freezing and freeze substitution. Protoplasma 147: 91-99.

Zundel, G. L. 1945. A change in generic name. Mycologia 37: 795-796. 